\title{
Cooperative MIMO multicell networks
}

\author{
Francesco Verde ${ }^{1 *}$, Yao-Win Peter Hong ${ }^{2}$, Dragan Samardzija ${ }^{3}$, Robert Schober ${ }^{4}$ and Zhifeng Tao ${ }^{5}$
}

Recently, the pressing desire to provide cost-effective solutions aimed at supporting high-throughput broadband wireless access with large-scale coverage has significantly changed the notion of the traditional cellular systems. Physical layer cooperation serves as an enabling technology for such a change. In latest cellular networks, neighboring infrastructure stations, such as base stations (BSs) or relay stations (RSs), share their communication resources to create virtual multiple-input multiple-output (MIMO) systems by means of distributed transmission and signal processing. Cooperative processing at the BSs promises to exceed the limits on spectral efficiency imposed by inter-cell interference, thereby allowing ever more aggressive frequency reuse patterns. On the other hand, cooperation between BSs and RSs, as well as among RSs, is expected to extend coverage and capacity of point-to-multipoint links between BSs and mobile stations in a highly economical fashion. However, to achieve such benefits in practice, numerous research challenges have to be still tackled. This special issue has come up with the intention of collecting cutting-edge research achievements in cooperative MIMO multicell systems.

For this special issue, we have received 35 manuscript submissions altogether. After a careful review process, 10 manuscripts have been accepted for publication, which cover the following topics for cooperative MIMO multicell networks: channel modeling, power control and scheduling, interference management, protocol design and performance analysis, cost deployment, simulation tools and testbeds. A brief introduction of all the accepted manuscripts is reported in the following.

In the manuscript "A Geometrical Three-Ring-Based Model for MIMO Mobile-to-Mobile Fading Channels in Cooperative Networks" by Talha and Pätzold [1], a stochastic narrowband MIMO mobile-to-mobile reference channel model is derived in relay-based cooperative networks and exact closed-form expressions for different

\footnotetext{
* Correspondence: f.verde@unina.it

${ }^{1}$ Department of Biomedical, Electronic and Telecommunication Engineering (DIBET), University of Naples Federico II, Naples, Italy Full list of author information is available at the end of the article
}

correlation functions are provided, under isotropic as well as non-isotropic scattering conditions. A stochastic simulation model is drawn from the reference model. It is shown that the cross-correlation functions of the simulation model closely approximate the corresponding ones of the reference model.

In the article "Adaptive Coordinated Reception for Multicell MIMO Uplink" by Lu et al. [2], power control and receive beamforming are jointly optimized with adaptive selection of multiple BSs to minimize the total transmit power, under individual signal-to-interferenceplus-noise ratio constraint per mobile station. To reduce the complexity in the large-scale cellular network, a suboptimal algorithm is derived, which exhibits a good trade-off between performance and complexity if the number of the pre-selected BSs is carefully chosen.

In the article "Virtual Cooperation for Throughput Maximization in Distributed Large-Scale Wireless Networks" by Abouei et al. [3], a distributed wireless network with $K$ links is con-sidered, where the links are partitioned into $M$ clusters each operating in a subchannel with bandwidth $W / M$. The power allocation and number of clusters of such a network are optimized for maximization of the throughput as the number of links approaches infinity.

In the article "Distributed Cooperative Precoding with Power Control for Cellular Systems with Correlated Antennas at the Receiver" by Vinosh Babu James et al. [4], the authors consider an analytical model for a multicell and multiuser system with receiver-side correlation. A distributed protocol for cooperation amongst the BSs is also proposed. System performance gains measured in terms of mean and cell-edge spectral efficiency values are reported.

In the article "Multimode Transmission in Network MIMO Downlink with Incomplete CSI" by Seifi et al. [5], a two-step scheduling algorithm for a cooperative multicell MIMO downlink is proposed: in the first step, joint user and mode selection is performed, whereas, in the second step, feedback and precoder design is carried out by only considering the users selected in the first step. The metric used to perform user and mode 
selection is based on an approximation of the ergodic user rates that involves the knowledge of incomplete channel state information (CSI) for any given location. A substantial reduction the feedback overhead is shown.

In the article "Interference Management Schemes for the Shared Relay Concept" by Panah et al. [6], several interference management strategies for relays shared by multiple cells are proposed. Since the relay functionality influences the total interference, both amplify-and-forward and decode-and-forward type relays are considered. Channel cancellation techniques are investigated for one-way and two-way relaying. It is shown that strategies based on two-way shared relaying with bi-directional channel inversion at the relay often perform best in terms of total system throughput while one-way techniques are promising when the relay power is low.

In the article "Cognitive Cooperation for the Downlink of Frequency Reuse Small Cells" by Akoum et al. [7], a cooperative diversity protocol is developed, which is coded over space, time, and frequency to improve the quality of service of mobile users. For this purpose a new two-phase cooperative protocol is proposed. In the first phase, each BS serves its own users in a dedicated frequency band and listens to the transmission of a neighboring BS in another frequency band. In the second phase, each BS transmits to its own users and the users of the other BS using both frequency bands and a suitable space-time code.

In the article "Performance Analysis of OFDM-based Decode-and-Forward Relay Network with Multiple Interferers over Rayleigh Fading Channel" by Tu et al. [8], the authors analyze an orthogonal frequency-division multiplexing (OFDM)-based decode-and-forward relay network impaired by frequency-selective Rayleigh fading and multiple uncorrelated equal power interferers. In particular, a unified closed-form expression of average symbol error rate for $M$-ary phase-shift-keying (M-PSK) modulation is derived and employed to study the performance of such systems.

In the article "Techno-economic Evaluation of Cooperative Relaying Transmission Techniques in OFDM Cellular Networks" by Moral et al. [9], an interdisciplinary approach is provided to evaluate the costs in the deployment of a $4 \mathrm{G}$ relay-assisted network in the 2.6 $\mathrm{GHz}$ band following a techno-economic methodology that departs from cell dimensioning based on spectral efficiency and outage capacity requirements. The presented results show significant benefits for operators, as well as reduction in the total radiated power.

In the article "Experimental Investigation of TDD Reciprocity-Based Zero-Forcing Transmit Precoding" by Zetterberg [10], the author describes an implementation on a wireless testbed of zero-forcing linear precoding when CSI at the transmitter is acquired by exploiting time-division-duplex (TDD) reciprocity. A self-calibration technique is described and significant improvements have been demonstrated over baseline schemes.

Finally, we would like to thank all the authors for their contribution to this special issue, the reviewers for their effort and time spent in reviewing the manuscripts, as well as the Editor-in-Chief Phillip Regalia and the Editorial Staff of the Hindawi Publishing Corporation for their support during the whole process.

\section{Author details}

'Department of Biomedical, Electronic and Telecommunication Engineering (DIBET), University of Naples Federico II, Naples, Italy ${ }^{2}$ Department of Electrical Engineering, Institute of Communications Engineering, National Tsing Hua University, Hsinchu, Taiwan ${ }^{3}$ Bell Labs, Alcatel-Lucent, Holmdel, NJ, USA ${ }^{4}$ Department of Electrical and Computer Engineering, University of British Columbia, Vancouver, Canada ${ }^{5}$ Mitsubishi Electric Research Laboratories (MERL), Cambridge, MA, USA

\section{Competing interests}

The authors declare that they have no competing interests.

Received: 1 April 2011 Accepted: 22 February 2012

Published: 22 February 2012

\section{References}

1. B Talha, M Pätzold, A geometrical three-ring-based model for MIMO mobile-to-mobile fading channels in cooperative networks. EURASIP J Adv Signal Process. 2011, Article ID 892871, 13 (2011)

2. X Lu, W Li, A Tlli, M Juntti, Adaptive coordinated reception for multicell MIMO uplink. EURASIP J Adv Signal Process EURASIP J Adv Signal Process. 2011, Article ID 195309, 11 (2011)

3. J Abouei, A Bayesteh, M Ebrahimi, AK Khandani, Virtual cooperation for throughput maximization in distributed large-scale wireless networks. EURASIP J Adv Signal Process. 2011, Article ID 184685, 19 (2011)

4. J Vinosh Babu James, B Ramamurthi, G Venkatraman, Distributed cooperative precoding with power control for cellular systems with correlated antennas at the receiver. EURASIP J Adv Signal Process. 2011 Article ID 706212, 12 (2011)

5. N Seifi, M Viberg, RW Heath Jr, J Zhang, M Coldrey, Multimode transmission in network MIMO downlink with incomplete CSI. EURASIP J Adv Signal Process. 2011, Article ID 743916, 13 (2011)

6. AY Panah, KT Truong, SW Peters, RW Heath Jr, Interference management schemes for the shared relay concept. EURASIP J Adv Signal Process. 2011, Article ID 269817, 14 (2011)

7. S Akoum, M Zwingelstein-Colin, RW Heath Jr, M Debbah, Cognitive cooperation for the downlink of frequency reuse small cells. EURASIP J Adv Signal Process. 2011, Article ID 525271, 11 (2011)

8. J Tu, Y Cai, W Yang, Performance analysis of OFDM-based decode-andforward relay network with multiple interferers over Rayleigh fading channel. EURASIP J Adv Signal Process. 2011, Article ID 202549, 15 (2011)

9. A Moral, J Vidal, J Prez, A Agustn, N Marina, A Hst-Madsen, Technoeconomic evaluation of cooperative relaying transmission techniques in OFDM cellular networks. EURASIP J Adv Signal Process. 2011, Article ID 507035, 23 (2011)

10. P Zetterberg, Experimental investigation of TDD reciprocity-based zeroforcing transmit precoding. EURASIP J Adv Signal Process. 2011, Article ID 137541, 10 (2011)

doi:10.1186/1687-6180-2012-41

Cite this article as: Verde et al:: Cooperative MIMO multicell networks. EURASIP Journal on Advances in Signal Processing 2012 2012:41. 